\title{
Sex matters: gender disparities in quality and outcomes of care
}

\section{Arlene S. Bierman MD MS}

$\infty$

See related article page $\mathrm{I} 5 \mathrm{I} 3$

Published at www.cmaj.ca on Nov. I4, 2007.

$\mathrm{I}$ $\mathrm{n}$ the not-too-distant past the $70-\mathrm{kg}$ male was the paradigm for medical practice and women were systematically excluded from clinical trials. In a relatively short period, sex- and gender-based analyses have contributed to an exponential growth in our knowledge about healthrelated differences between women and men. Advances in molecular biology and genomics have found that differences between the sexes extend to the cellular level. ${ }^{1}$ Sex differences in pathophysiology and phamacokinetics increasingly have important implications for clinical practice. Clinical epidemiology, health services research, outcomes research and the social sciences are all making profound contributions to our understanding of sex and gender differences in health and disease. Women and men differ not only in patterns of illness and disease risk factors, but also in their social contexts. Consequently, they have different experiences with health care, including differences in access, quality and health outcomes. Although sex and gender differences have been most extensively studied in cardiovascular care, ${ }^{2,3}$ the more we examine other areas, the more differences we find.

In a large retrospective cohort study published in this issue of CMAJ, Fowler and colleagues ${ }^{4}$ report on important disparities associated with sex that they found in intensive care use and outcomes between men and women. Specifically, older women (aged 50 or older) were less likely than older men to be admitted to the intensive care unit (ICU) or to receive life-saving interventions such as mechanical ventilation, and more likely to die when admitted because of a critical illness. Despite similar severity of illness between women and men upon ICU admission, women had shorter stays in the ICU but longer overall stays in hospital. By linking data from the Canadian Critical Care Research Network registry to administrative data, the authors were able to control for a large number of potential confounders, including demographic characteristics, principal diagnosis, comorbidities and illness severity (APACHE [Acute Physiologic and Chronic Health Evaluation] scores). Because these findings are consistent with those from prior studies that suggest the existence of sex and gender disparities in ICU care, we can have a high level of confidence that, despite the limitations

\section{Key points of the article}

- Important sex and gender disparities in intensive care use and outcomes have been identified, as they have in many other areas of clinical practice. Critically ill women 50 years and older were less likely than critically ill men to be admitted to an intensive care unit (ICU) and to receive potentially life-saving interventions, and they were more likely to die in ICU or in hospital.

- It is likely that patient-level, provider-level and system-level factors contribute to observed sex and gender disparities in quality and outcomes of care for critical illness.

of the study by Fowler and colleagues, the sex disparities they identify are real. Particularly troubling is the finding of sex differences in mortality.

Other recent studies have found women to be at increased risk for receiving suboptimal care for serious illness. For example, Birnie and colleagues ${ }^{5}$ found that women were less likely than men to receive implantable cardiac defibrillators after an out-of-hospital cardiac arrest. Two additional studies suggested that women were less likely than men to receive implantable cardiac defibrillators when indicated for congestive heart failure. ${ }^{6,7} \mathrm{Kaul}$ and colleagues ${ }^{8}$ found that women presenting to the emergency department with coronary syndromes were less likely than men to be admitted to an acute care hospital and to receive coronary revascularization procedures. Yet another study found that older women were at increased risk, when compared with men, for receiving potentially inappropriate medications. ${ }^{9}$

What the study by Fowler and colleagues cannot tell us is what causes these disparities, or what we should do to close these gaps. It is likely that patient-level, provider-level and system-level factors all contribute to observed sex disparities in quality and outcomes of care for critical illness. Although population-based mortality is lower overall among older

Arlene Bierman is the Ontario Women's Health Council Chair in Women's Health, Faculties of Medicine and Nursing, University of Toronto. She is also with the Li Ka Shing Knowledge Institute, St. Michael's Hospital, Toronto, Ont. 
women than among older men, ${ }^{10}$ as Fowler and colleagues point out, there may be plausible biological explanations for the observed differences in mortality. There may also be sex differences in patient preferences for care and end-of-life decision-making. However, we cannot exclude gender bias on the part of providers. In addition, there may be an important interaction between sex and age bias, because Fowler and colleagues found important differences among women aged 50 or older. Finally, health system design and organization that fails to meet the needs of older women may also be a contributing factor.

The study by Fowler and colleagues, when placed in the context of the large body of literature documenting sex and gender differences in quality and outcomes of care, has a number of important implications for practice, research and policy. In 200I, in a comprehensive report entitled "Exploring the Biological Contributions to Health: Does Sex Matter?" the Institute of Medicine concluded that "sex should be considered when designing and analyzing studies in all areas and at all levels of biomedical and health-related research." ${ }^{1} \mathrm{~A}$ recent report on gender and equity in health by the World Health Organization Commission on the Determinants of Health observed that "taking action to improve gender equity in health ... is one of the most direct and potent ways to reduce health inequities and ensure effective use of health resources." 11 Since 1994 the US National Institutes of Health has mandated the inclusion of women in clinical trials. The Canadian Institutes of Health Research has a policy supporting sex- and gender-based analysis. Yet, women in general, and older women in particular, are still underrepresented in clinical trials. Even when included in appropriate numbers to derive reliable estimates, sex- and gender-based analyses are not routine. ${ }^{12}$

If we are to optimize health outcomes for women and men, 3 fundamental strategies need to be implemented. First, sex- and gender-based analyses should become routine in all health research, including basic sciences, social sciences, clinical epidemiology, health services and outcomes research. It can no longer be acceptable to just control for sex; at a minimum, it is necessary to assess whether sex, gender or both are factors in study outcomes. When differences are found, further analyses should be required to explore the contributing factors. Second, if we are to improve to quality of care for women and men, quality indicators need to be sex-stratified in order to assess whether sex and gender disparities in performance are present. When disparities are identified, targeted interventions to reduce and ultimately eliminate these disparities should be developed. ${ }^{13}$ In January 2008, the Project for an Ontario Women's Health Evidence-based Report Card (POWER) will release a report on a comprehensive set of quality indicators stratified by sex. This report will contribute to our knowledge of where sex and gender disparities in health care quality are an issue. ${ }^{14}$ Third, it is time to move beyond descriptive studies and, instead, to give priority to research that elucidates the underlying pathways leading to ob-
Strategies to optimize health outcomes for women and men

- Sex- and gender-based analyses should be routine in all health research, including basic sciences, social sciences, clinical epidemiology, health services and outcomes research.

- To improve the quality of care for women and men, quality indicators should be sex-stratified to assess whether sex and gender disparities in performance are present. When disparities are identified, targeted interventions to reduce these disparities should be developed.

- It is time to give priority to research that elucidates the underlying pathways leading to sex and gender disparities and that develops the evidence for effective interventions to achieve sex and gender equity in health and health care.

served sex and gender disparities and that develops the evidence for effective interventions to achieve sex and gender equity in health and health care. Vive la différence!

Competing interests: None declared.

\section{REFERENCES}

I. Wizemann T, Pardue M. Exploring the biological contributions to health: Does sex matter? Washington: National Academy Press; $200 I$.

2. Bird CE, Fremont AM, Bierman AS, et al. Does quality of care for cardiovascular disease and diabetes differ by gender for enrollees in managed care plans? Womens Health Issues 2007;17:131-8.

3. Pilote L, Dasgupta K, Guru V, et al. A comprehensive view of sex-specific issues related to cardiovascular disease. CMAJ 2007;176:SI-44.

4. Fowler RA, Sabur N, Li P, et al. Sex- and age-based differences in the delivery and outcomes of critical care. CMAJ 2007; I77:1513-9.

5. Birnie DH, Sambell C, Johansen H, et al. Use of implantable cardioverter defibrillators in Canadian and US survivors of out-of-hospital cardiac arrest. CMAJ 2007; I77:4I-6.

6. Curtis LH, Al-Khatib SM, Shea AM, et al. Sex differences in the use of implantable cardioverter-defibrillators for primary and secondary prevention of sudden cardiac death. JAMA 2007;298:1517-24.

7. Hernandez AF, Fonarow GC, Liang L, et al. Sex and racial differences in the use of implantable cardioverter-defibrillators among patients hospitalized with heart failure. JAMA 2007;298:1525-32.

8. Kaul P, Chang W, Westerhout C, et al. Differences in admission rates and outcomes between men and women presenting to emergency departments with coronary syndromes. CMAJ 2007:177:1193-99.

9. Bierman AS, Pugh MJ, Dhalla I, et al. Sex differences in inappropriate prescribing among elderly veterans. Am J Geriatr Pharmacother 2007;5:I47-6r.

Io. Walter LC, Brand RJ, Counsell SR, et al. Development and validation of a prognostic index for I-year mortality in older adults after hospitalization. JAMA 200I;285:2987-94.

II. Sen G, Östlin P, George A. Unequal, unfair, ineffective and inefficient: gender inequity in health: Why it exists and how we can change it [final report to the WHO Commission on Social Determinants of Health]. Stockholm: Karolinska Institutet 2007. Available: http://genero.sespas.es/pdf/WGEKN\%20FINAL\%20REPORT.pdf (accessed 2007 Oct 22).

I2. Geller SE, Adams MG, Carnes M. Adherence to federal guidelines for reporting of sex and race/ethnicity in clinical trials. J Womens Health (Larchmt) 2006;15:II23-3I.

I3. Bierman AS, Clark JP. Performance measurement and equity. BMJ 2007;334:1333-4

I4. POWER Study. Project for an Ontario Women's Health Evidence-based Report Card. Available: www.powerstudy.ca (accessed 2007 Oct 22).

Correspondence to: Dr. Arlene S. Bierman, Li Ka Shing Knowledge Institute, St. Michael's Hospital, 30 Bond St., Toronto ON

M5B IW8; fax 4I6 864-6057; arlene.bierman@utoronto.ca 